vol. 24 - $n^{\circ} 3 \mid 2008$

Numéro ouvert

\title{
Population mobile dans les grandes entreprises: compétences acquises et coûts humains
}

Laura Gherardi

\section{(2) OpenEdition \\ 12 Journals}

Édition électronique

URL : https://journals.openedition.org/remi/4847

DOI : $10.4000 /$ remi.4847

ISSN : $1777-5418$

Éditeur

Université de Poitiers

\section{Édition imprimée}

Date de publication : 1 décembre 2008

Pagination : 191-207

ISBN : 978-2-911627-50-7

ISSN : 0765-0752

\section{Référence électronique}

Laura Gherardi, «Population mobile dans les grandes entreprises : compétences acquises et coûts humains ", Revue européenne des migrations internationales [En ligne], vol. $24-n^{\circ} 3$ | 2008, mis en ligne le 31 août 2010, consulté le 16 avril 2022. URL : http://journals.openedition.org/remi/4847 ; DOI : https://doi.org/10.4000/remi.4847 


\section{Population mobile dans les grandes entreprises : compétences acquises et coûts humains}

\section{Laura GHERARDI* et Philippe PIERRE**}

"Les insulaires d'Albion apportent avec eux un fluide particulier que j'appellerai le fluide britannique, et au milieu duquel ils voyagent, assez peu accessibles à l'atmosphère des régions qu'ils traversent que la souris au centre de la machine pneumatique. (...) C'est parce que l'air extérieur n'a pas de prise sur eux ; c'est parce qu'ils marchent, boivent, dorment et mangent dans leur fluide, comme dans une cloche de cristal de vingt pieds, et au travers de laquelle ils regardent en pitié les cavaliers que le vent défrise et les piétons dont la neige endommage la chaussure. (...) Ce n'est pas leur personne, c'est leur garde-robe qui voyage, et l'homme n'est que l'occasion du porte manteau, le véhicule de l'habillement"

(Sand, dans un voyage effectué en Suisse et à Chamonix en 1836, citée par Geoffroy, 2005).

L'intensification des voyages, des migrations, des mobilités pour les plus privilégiés mais aussi les phénomènes de déréglementation des marchés, les stratégies des grandes firmes et l'introduction d'innovations technologiques rétrécissant le temps et l'espace (Giaccardi et Magatti, 2003), confèrent certainement une version inédite des rapports entre les êtres. Ces phénomènes nourrissent aujourd'hui, au sein des sociétés occidentales, une démultiplication des objets et des terrains d'études liés à la mobilité

* Doctorante en sciences sociales (École des Hautes Études en Sciences Sociales, Paris, et Université Cattolica del Sacro Cuore, Milan).

** Consultant. Chercheur associé au sein du Laboratoire Interdisciplinaire pour la Sociologie Économique (LISE/CNRS). www.philippepierre.com 
géographique dans la mondialisation (Harvey, 1989 ; Urry, 2000) : tourisme de masse, utilisation à distance des nouvelles technologies, des médias, des diasporas, des nonlieux, de la transnationalisation des religions et des cultures...

La mobilité géographique d'ordre professionnelle, brièvement définie comme la mobilité d'un individu dans l'espace physique pour des raisons de changement de lieu de travail, se décline le plus souvent en deux types : les migrations, qui concernent des changements définitifs d'espaces de vie, et les circulations qui évoquent des mouvements « temporaires » comme l'expatriation.

Un phénomène récent de substitution, dans les pays riches, des formes les plus irréversibles de mobilité telles que la migration ou la mobilité résidentielle de longue durée s'opère vers des formes de multi-résidence, de mobilités quotidiennes, pendulaires ou de courts voyages (Martin, Metzger et Pierre, 2003).

Dans le monde du travail des grandes entreprises, notamment pour des raisons budgétaires, se fait jour un mouvement de diversification des formes de mobilité internationale pour certains dirigeants (top management) et pour des cadres mobiles que cet article s'attachera à distinguer (Black et Gregersen, 1999 ; Berthoin Antal, 2000). Ce mouvement à trait à l'amélioration des transports à grande vitesse, avions et trains, aux pratiques nouvelles de « commuting » (comme le fait de travailler à Londres la semaine et vivre à Paris le week-end) et de mobilités d'une à deux jours sur des aires géographiques de plusieurs centaines voire milliers de kilomètres.

Dans une première étape de cet article, nous pouvons isoler, dans les plus grandes entreprises françaises, les salariés mobiles issues de la maison mère (expatriés), les cadres recrutés localement du pays parent, les chargés de missions brèves, les salariés locaux, les salariés locaux temporairement dans un pays tiers, les impatriés (salariés locaux au siège du pays parent) ainsi que certains grands mobiles (« cosmopolites ») (Pierre, 2003).

Dans une seconde étape, nous pouvons distinguer des mobilités d'expertise (visant à transférer un savoir-faire technique ou procédural), des mobilités d'appartenance à une organisation (visant davantage à former et fidéliser de jeunes cadres « à potentiel »), des mobilités de gouvernance (visant à diriger une filiale et à contrôler un certain nombre d'intérêts patrimoniaux) (Harzing, 2001) ainsi que la multiplication d'expériences de travail simultanées dans plusieurs pays quand le salarié appartient à une entité ayant des implantations dans différents pays (plates-formes d'achats, laboratoires de recherches, usines...) (de Wenden, 2001).

Dans la plupart des grandes entreprises françaises que nous avons pu étudier depuis quinze ans, la majorité des salariés mobiles font de plus en plus partie de groupes «projets » (Schweiger, Atamer et Calori, 2003) qui s'ajoutent à leurs missions opérationnelles et qui posent des questions concrètes de coordination du travail des équipes comme d'appréciation des performances (Hedlund, 1994 ; Birkinshaw et Hood, 1998 ; Gupta et Govindarajan, 2000). Il n'est plus rare de lier un travail principal au siège de la société, des déplacements dans de nombreuses filiales, des 
réunions régionales ou internationales, avec un lieu de résidence familiale dans un autre pays du fait des études spécifiques des enfants ou du travail du conjoint. De nouvelles formes d'intermittence de la vie familiale, de bi-résidentialité se construisent par rapport à l'intérêt du travail et viennent aussi reconfigurer les conditions de vie dans les foyers familiaux des salariés mobiles (Vignal, 2005 ; Bertaux-Wiame et Tripier, 2006). Les appellations récentes de "cadres mobiles », «transfrontaliers », « international business travellers », «road warriors», « globe-trotters » ou «frequent fliers» témoignent parfois de formes nouvelles de mobilité («management by flying about») pouvant aller jusqu'à une mobilité dite «virtuelle » et permise par l'usage des nouvelles technologies de l'information et de la communication ${ }^{1}$. Une interactivité réelle ou supposée des échanges de façon synchrone se mêle de plus en plus à un exercice professionnel asynchrone (avec des outils de messagerie tels que le chat ou le forum, l'automatisation des processus, la production à distance...) (Cohen, 2005).

\section{ASPECTS MÉTHODOLOGIQUES}

Une enquête réalisée par la Conférence des grandes écoles, en 2000, indiquait que $10 \%$ des diplômés d'écoles françaises d'ingénieurs, 12 à $15 \%$ de diplômés d'écoles de commerce et $25 \%$ des diplômés d'HEC commençaient leurs carrières à l'étranger ${ }^{2}$. On ne dispose pourtant que de peu d'études sur ces acteurs agissant dans le développement du capitalisme dans sa phase de réorganisation spatiale la plus massive depuis les origines. Une approche compréhensive des pratiques de mobilité géographique est donc à promouvoir à l'heure des entreprises mondialisées. Elle exige d'analyser la généalogie des pratiques «mobilitaires », d'en sonder les systèmes de valeurs, les technologies, les usages du corps, des pratiques de communication de ceux qui les vivent (De Bettignies et Evans, 1977).

Nous nous proposons de faire un pas en cette direction, dans cet article, en traçant les configurations « spatio-temporelles » des différentes formes de mobilité que nous avons repérées au cours de notre travail d'enquête et qui ne sont pas sans liens avec les coûts humains cachés que la mobilité fait aussi peser sur les individus. Notre travail d'enquête est basé sur une quarantaine d'entretiens ${ }^{3}$ avec des cadres expatriés,

1 L'expatriation virtuelle vise à pallier l'envoi d'un ou plusieurs cadre(s) dans chacune des filiales étrangères, en déléguant certaines tâches internationales à un salarié, basé à un poste fixe pour une zone géographique déterminée et ayant à disposition un équipement multimédia sophistiqué (vidéoconférence, communication via Internet et messageries internes...).

2 Les organes de presse économique encouragent l'effet d'attraction d'un « capital de mobilité » à se constituer pour tout jeune cadre et la possibilité, par les nouvelles technologies (Blackberry, Personal development assistant...), de profiter d'interactions qui ne dépendent plus de la seule proximité physique. L'accumulation du capital symbolique, d'opportunités renforcées de faire carrière et surtout d'acquérir les bonnes conditions d'employabilité pour plus tard, apparaît comme un déterminant du choix de partir à l'étranger.

3 Notre enquête se base sur un échantillon de 43 personnes admettant la répartition suivante : - 20 entretiens semi-directifs pour des cadres intermédiaires vivant une mobilité trans- 
des cadres internationaux et des dirigeants (top management) appartenant à trois multinationales d'origine française et sur un ensemble de données recueillies sur un mode d'observation participante des auteurs. Pour répondre à ces questions, il nous a fallu introduire d'autres types de mobilité que la mobilité géographique qui nous est apparue, en soi, un indicateur trop grossier du pouvoir à être mobile détenu par ces individus.

Dans leurs travaux, Chiapello et Boltanski (1999) distinguent, au sein d'une sphère capitaliste professionnelle appréhendée de manière globale, dans un monde d'entreprises en réseaux, les « grands/mobiles/globaux », les « petits/rigides/locaux » et les flexibles :

Schéma 1

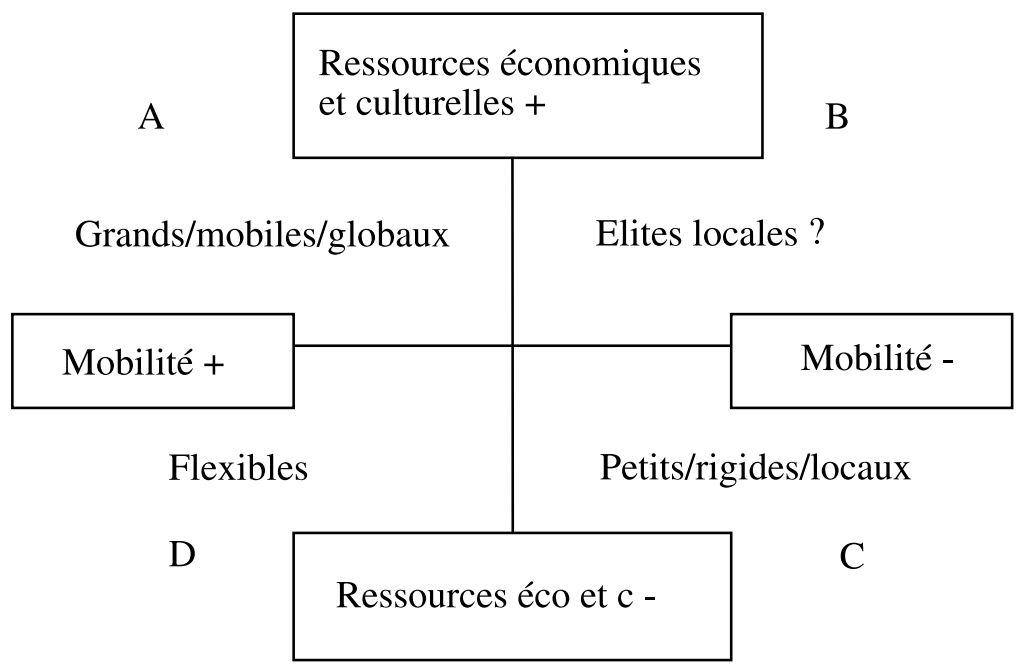

nationale (cadres impatriés en France, expatriés et internationaux) et 7 entretiens pour les membres de la direction (du Directeur Général aux Seniors Vice-présidents) dans la même firme multinationale, dont le siège est à Paris et que nous appelons «multinationale 1 ».

- 12 entretiens auprès de managers appartenant à deux autres firmes multinationales, de la même taille de chiffre d'affaires que la première, qui ont aussi leurs sièges à Paris et qui n'ont pas effectué de coupes aux dépenses en voyages d'affaires dans les dernières cinq années, que nous appelons «multinationale 2 » et «multinationale 3 ». Nous distinguons 8 entretiens pour une population appartenant au « middle management» et 4 personnes au «top management». - 4 entretiens auprès d'informateurs privilégiés dans les métiers du commerce et du contrôle de gestion. La durée de ces entretiens a toujours été d'une heure à trois heures. Pour ce qui concerne les développements du paragraphe 1.1, nous nous sommes basé aussi sur les résultats des travaux de P. Pierre issus d'une enquête dans une firme multinationale française du secteur pétrolier 2003) ; pour ce qui concerne le paragraphe 1.3, nous avons aussi pu avoir accès à plusieurs planning de mobilités de cadres et dirigeants rencontrés sur trois années calendaires. 
L'échantillon de notre article est fondé principalement par des représentants des grands mobiles globaux (A) pour lesquels n'existent que peu d'études sociologiques de type empirique (Wagner, 1998 ; Wagner, 2003 ; Agard, 2004). Les analyses sociologiques sur la mobilité géographique, qui à l'échelle planétaire parlent de migrations et à l'échelle métropolitaine de phénomène d'exclusions ou de marginalité, se concentrent, en effet, actuellement sur des groupes discriminés de la partie basse de notre schéma. Rares sont les études qui font état des différentiels de pouvoir liés à la hiérarchie interne des entreprises face aux logiques de mobilité des effectifs. C'est au prix de telles omissions que perdure peut-être l'idée d'élite homogène composée de citoyens du monde qui se reconnaissent et agissent de concert. L'homme mobile et léger - privé de toute attache pesante - assumerait, dans certaines rhétoriques du management interculturel, les traits d'un nouvel explorateur culturel, d'un « hommejet », féru de technologie, prêt à apprendre toute sa vie, doté d'un esprit pionnier, souvent mobile également dans sa vie personnelle et affective comme dans le registre de ses engagements matrimoniaux. La mobilité géographique requise par l'entreprise y figure comme la réalisation d'un destin heureux choisi par des sujets dont la nature est curieuse, ouverte aux autres, en un mot, «vagabonde » et « efficace ». Dans un processus entretenu de dévalorisation constant de la stabilité territoriale, la nature de ces nouveaux explorateurs les opposerait aux collègues sédentaires qui n'ont pas le courage de relever le «challenge » de la mobilité.

La mobilité géographique des effectifs des plus grandes entreprises françaises est donc posée comme source d'épanouissement personnel et professionnel. C'est précisément ce lien entre mobilité et épanouissement personnel qui est remis en cause par une partie de notre échantillon.

En fait, à côté de ceux dont l'unique regret est celui de n'être pas partis avant et qui soulignent le caractère enrichissant de la mobilité, d'excitation continuelle, nous avons rencontré d'autres personnes qui parlent de leur mobilité comme d'une contrainte, comme d'un poids ou, plus rarement, comme d'un piège. Les différentes articulations de coûts dénoncés par les cadres expatriés et par le top management ne sont pas sans relation avec les différentes configurations « spatio-temporelles » dans lesquelles ces effectifs, qui n'épuisent pas l'ensemble de la population mobile de l'entreprise $^{4}$, sont insérés. Parler de configurations « spatio-temporelles », c'est-à-dire

4 Il nous paraît nécessaire de préciser que les deux groupes sur lesquels nous nous concentrons ici, c'est-à-dire le management intermédiaire et le «top management », n'épuisent pas l'ensemble de la population mobile de l'entreprise. Nous avons choisi de nous focaliser sur ces deux groupes puisque la littérature propre au management interculturel, vise à niveler les différences notamment entre le middle management qui en est la cible, lui fournissant des arguments pour en motiver les déplacements géographiques requis par l'entreprise, et le top management qui en est le groupe de référence. Dans l'entreprise que nous avons appelée " multinationale 1 », on peut rencontrer, par exemple, des techniciens à haut niveau de spécialisation sur une ou plusieurs machines/systèmes de production et qui se déplacent, à une échelle planétaire pour le temps nécessaire à la réparation ou à l'entretien, ou encore des techniciens qui se déplacent afin d'effectuer des expertises concernant l'état des outillages d'une entreprise étrangère. On croise aussi des experts et consultants internationaux qui se 
de formes de mobilité qui se différencient selon leur durée, selon leurs motivations et leurs directions et selon les coûts pesant sur les salariés, revient à contester l'idée selon laquelle le personnel qualifié de la multinationale, qui effectue des déplacements géographiques à échelle internationale, partage une même mobilité considérée comme le déplacement d'un sujet d'un point à un autre de l'espace géographique.

Les rhétoriques managériales qui détachent le cadre mobile de l'organisation dans laquelle il est inséré et de la position hiérarchique qu'il y occupe, apparaissent très sommaires dès lors que l'on demande à ces effectifs internationaux de l'entreprise de détailler leurs déplacements. En particulier, dès nos premiers entretiens avec les cadres et dirigeants mobiles dont les déplacements géographiques à échelle transnationale s'entrecroisent dans le siège parisien de la multinationale considérée, deux variables sont d'abord parues distinctives : la direction des déplacements effectués par le sujet — entre les différents établissements répartis de l'entreprise et/ou vers des partenaires extérieurs à l'organisation liées aux raisons d'être des voyages — et la durée de ces déplacements, c'est-à-dire la variable temps des missions hors du pays d'origine. Si l'on considère ces deux paramètres, la mobilité des individus ne se réduit en rien à une ligne, mais s'ébauche en termes de configurations « spatio-temporelles » variées, c'està-dire récurrentes et distinctes, venant constituer autant de formes de mobilité géographique pour notre échantillon ${ }^{5}$. Nous en proposons quatre dans cet article : expatriation d'expertise ou de conquête de marché, mobilité réticulaire à base unique, double mobilité scalène et circulation du top management à mobilité externe.

\subsection{Savoir résider. Cadres expatriés à la frontière de plusieurs cultures et appartenances ?}

\subsubsection{Configuration « spatio-temporelle » 1 : expatriation d'expertise ou de conquête de marché}

Dans cette configuration, l'expatriation consiste en un déplacement du siège du pays parent vers une filiale en terre étrangère pour une durée habituelle de 2 à 5 ans. Les expatriés, sélectionnés principalement sur la base d'un critère de fidélité à la

déplacent à l'intérieur de circuits transnationaux et qui, n'étant pas parmi les effectifs du personnel permanent, transitent dans l'entreprise le temps d'une consultation. On peut également recenser des recruteurs, des commerciaux et des responsables de secteurs/ coordinateurs de zones qui se déplacent à des échelles spatiales différentes, ou bien des effectifs, profitant des nouveaux transports à grande vitesse, en « commuting » (par exemple des chercheurs qui se déplacent par intermittences régulières - le plus souvent, sur une semaine - entre deux ou trois laboratoires de la même multinationale répartis dans des pays différents d'un même continent).

5 Nous sommes bien loin du déterminisme. Nous avons déjà souligné comment, face aux effets d'une « impotence sociale temporaire » à l'étranger, selon les termes de Jean Yves Agard, pesant sur le middle management mobile géographiquement, certains vont réussir à composer avec des cultures différentes du pays d'origine, et offrir au moins cinq différentes stratégies identitaires en réponse (Pierre, 2005). 
maison mère ${ }^{6}$ et d'un potentiel avéré de développement professionnel, peuvent être envoyés suite, notamment, à une fusion ou à une acquisition d'une entreprise étrangère (quand le management souhaite en modifier l'équipe de direction, l'organisation du travail ou les procédures utilisées) (Cerdin, 2007) ou bien à la suite de l'ouverture d'une filiale à l'étranger quand on confie à un fidèle de l'entreprise le devoir de prospecter et conquérir un nouveau marché (Pierre, 2003).

Au cours des entretiens réalisés avec des expatriés, nous sont apparus des problèmes vécus de cohérence entre milieu d'accueil et univers de valeurs et de normes incorporées. "Faire l'expérience de la mobilité internationale, c'est souvent voir disparaître les points de repère culturels habituels qui balisent et simplifient la vie en société dans son pays d'origine (...) avoir le souci exagéré de la propreté et du contacte physique, se préoccuper de manière quasi-obsessionnelle des problèmes de nourriture, d'eau potable, de literie » constate un Responsable de formation en charge de séminaires de préparation à la vie expatriée dans une multinationale française du secteur pétrolier.

Se questionner sur la nature des apprentissages dans un contexte de confrontation culturelle intense et sur l'élaboration de la socialisation d'un cadre qui vit l'expérience d'une mobilité internationale renouvelée nous a invité à penser ensemble mobilité géographique, subjectivité et construction des identités. Un entre-deux de l'ici du pays d'origine et de l'ailleurs du pays d'expatriation est présent dans les discours de ces cadres, incitant le chercheur à préciser la nature de l'ancrage socio-historique vécu, à tenter d'objectiver les capitaux académiques, familiaux et sociaux. L'expatriation, aussi bien que l'impatriation, font partie de ces expériences qui déplacent le sujet de ses habitudes et le propulse dans un milieu où il est contraint de devoir confronter différents codes et s'adapter en reliant les lieux, les partenaires de sa présence et aussi ceux de sa mémoire. Ramos $(2006: 58)^{7}$ propose la notion d'ancrage « à disposition » et d'ancrages " à distance » (par exemple, le maintien d'une maison dans le pays d'origine) et parle de l'existence d'un « ailleurs significatif » qui conserve souvent davantage de force que l'existence d'un bien concret.

Pour ces cadres, s'opère souvent comme une inversion des lieux de socialisation repérée par Agard. L'entreprise devient un refuge, un lieu de routine et d'évitement du monde extérieur, alors que la famille devient un lieu de conflits. L'autonomie est mise à mal pour ses proches : interactions sociales dans une nouvelle langue, rupture d'avec les repères familiers, indisponibilité des affaires personnelles bloquées parfois à la douane plus qu'on ne l'envisageait... La chute brutale du capital social

6 Tendance à nommer des hommes et des femmes qui présentent un profil culturel et national homogène. "Alors que les entreprises étudiées réalisent en moyenne $80 \%$ de leur chiffre d'affaires hors d'Allemagne, qu'elles y emploient $61 \%$ de leurs salariés et qu'elles y détiennent $53 \%$ de leurs actifs, le degré d'internationalisation de leurs dirigeants ne dépasse pas $23 \%$. Les dirigeants sont donc significativement moins internationaux que les firmes qu'ils dirigent $\gg$ constate Stefan Schmid (2006).

7 « Certains souvenirs olfactifs, auditifs, sensoriels font aussi office d'éléments d'ancrage au sens où un son, une odeur, tout comme un objet, peuvent constituer un repère biographique qui ramène l'individu à un lieu et un temps donné ». 
relationnel n'est pas compensée par le capital symbolique offert par l'expatriation (le capital $^{8}$ est comme « inconvertible »). Agard distingue un « savoir circuler », c'est-àdire une aisance personnelle et un savoir-faire lié aux techniques de déplacement qu'il distingue d'un «savoir résider » où les ressources conférées par l'épouse, la famille et la communauté de pairs (compatriotes, amis, collègues, membres des mêmes associations de bienfaisance...) sont premières.

Les rhétoriques managériales traitent peu de ces difficultés et préfèrent valoriser la notion de « compétence culturelle » (Kealey, 1990) face à celle, réifiée, de « choc culturel ». Quand ces dissonances culturelles et identitaires sont mentionnées, tout au plus apparaissent-elles comme une rupture dans les routines de la vie que doit venir compenser une forte identification à la culture de l'entreprise. Dans nos enquêtes, nous rejoignons les observations de Wagner pour qui « l'international n'abolit pas le national » au sens que, dans les espaces transnationaux, la nationalité d'origine est un élément qui «stratifie » la population. Ainsi, l'espace transnational de travail des cadres reste fortement hiérarchisé et la couleur globale n'efface pas la couleur locale.

\subsection{Savoir circuler. Cadres internationaux, entre éloge et dénoncia- tion de leurs mobilités}

\subsubsection{Configuration « spatio-temporelle » 2 : mobilité réticulaire à base unique}

Nous appelons « internationaux » les cadres dont la mobilité, différemment des populations expatriées, requiert des déplacements fréquents et généralement d'une durée de quelques jours à quelques semaines, dans des pays étrangers différents. Ces cadres internationaux peuvent exercer des fonctions d'animation de projets transversaux en cours, de supervision sur un territoire géographique (une direction logistique ou commerciale d'une zone de 5 pays européens, par exemple) ou de coordination d'équipes multinationales — qui ne leur sont pas nécessairement rattachées hiérarchiquement. Le trait commun de ces cadres est : bouger à partir d'une base unique (le siège ou les sièges de l'entreprise généralement). Le nombre des déplacements, aussi bien que leur direction vont varier d'un cadre international à un autre et, pour un même individu, varier sur des périodes calendaires différentes, puisque les déplacements mêmes, dans cette configuration, suivent souvent les projets internationaux lancés ou suivis.

Certains cadres internationaux, comme ce responsable de projet formation et développement marketing international, parleront de « double mobilité » — selon l'expression même de l'interviewé : "Maintenant je vis une double mobilité, comme je ne suis pas chez moi en Espagne et, qu'en plus, je me déplace de Paris à l'étranger pour les besoins de ma mission ».

8 Nous maintenons ici le terme capital dans un sens purement analogique d'ensemble de ressources et de compétences. 


\subsubsection{Configuration « spatio-temporelle » $3:$ double mobilité scalène (en référence aux triangles dont les trois côtés sont de longueurs différentes)}

Dans cette configuration, la mobilité géographique s'exerce majoritairement dans une autre entité à distance (la majeure partie de l'année) sans exclure les déplacements courts en d'autres pays ou entités de l'entreprise.

Une grande partie du middle management que nous avons rencontré fait état d'un distinguo entre une mobilité choisie et une mobilité imposée, entre une mobilité vécue en tant que ressource (un « accélérateur » de carrière) et une mobilité en tant qu'impératif (un « investissement » nécessaire et qu'il faut devoir faire).

Ainsi, après nous avoir déclaré être "fait pour voyager, pour explorer " et donc «à l'aise partout», ce jeune cadre nous a aussi dit que «voyager c'est angoissant. Dès que je suis parti à l'international, j' ai grossi et suis devenu méconnaissable. Quand on vit ailleurs il faut se réconforter, il faut que tu te fasses plaisir et habituer ton corps à résister aux décalages ».

En un temps court de l'entretien, ce sujet se déclare « espagnol à $100 \%$ » et, quelques minutes plus tard, dit se sentir « citoyen du monde, à l'aise partout », « qu'il est chez lui à Paris et qu' il rentre chez lui à Madrid un week-end par mois parce qu' il a besoin de retrouver les endroits qu'il aime, auxquels il appartient, parce que c'est en Espagne qu'il retrouve tous ses repères, sa famille, ses amis qu'il connaît depuis qu'il est petit, son chien, sa grand-mère, le boulanger à côté et qui lui réserve son pain, des choses bêtes qui rassurent ». Nous analysons ces discours comme une réappropriation positive de leurs origines et aussi, face à un environnement qui peut être perçu comme menaçant, une capacité des acteurs à déclencher des procédures de contrôle des désignations qui les assaillent. En cela, l'identité du cadre international se définit bien à la fois par ce qui est subjectivement revendiqué et ce qui est socialement accordé. À l'opposé de l'immigré de faible condition sociale, contraint souvent à l'assimilation forcée, les cadres mobiles expriment un souci de réalisation de soi qui n'est pas une simple défense narcissique contre un environnement sur lequel les individus ont peu de prises. Des cadres internationaux interviewés font état de ces rapports doubles, «pulsatoires », à la variable nationale/domestique comme lieu des origines ( "J'ai eu plaisir à faire mon "coming out” quand j"ai pu témoigner, moi jeune femme blanche originaire d'Afrique du Sud, en arborant une broche représentant le continent africain, mes origines pendant toute une semaine de formation dans mon entreprise »). "Moi qui suis né en France, et qui en a hérité la nationalité, je me sens l'âme irlandaise au moment de la Saint Patrick le 17 mars. Ma famille aussi. Je ne sais pas pourquoi parce que l'on a passé seulement 16 mois à Dublin en expatriation et l'on rentrait presque tous les deux mois en France. À la troisième génération, du côté de ma femme, il y a une branche irlandaise. Quand il rejoint, chez de proches amis, pendant les vacances, la terre de ses lointains ancêtres, mon fils laisse à son poignet le décalage d'une heure de son voyage en Irlande. Il sait qu'il endossera, quand il rentrera, le rôle d'ambassadeur temporaire du pays au trèfle à trois feuilles, de Johnatan Swift et de James Joyce ». 
Dès nos premiers entretiens, le rôle joué par le «capital international » transmis par la famille d'origine des personnes étudiées, est apparu important, comme nous l'explique ce cadre international, fils de militaire : "j' ai ça dans le sang. Mon père à vécu en Maroc toute son enfance, car mon grand-père y avait des usines de savon. Je suis né en Algérie. De 5 à 10 ans, j'ai vécu au Colorado sans revenir jamais en France. Je parlais mieux anglais que français, puis le lendemain de mai 68, je me suis trouvé à Chambéry dans les Alpes. J'étais accueilli comme un Américain, un capitaliste fils de militaire dans un milieu ouvrier... après ça, je suis parti à Londres 3 ans. J'étais invité à des boums du mouvement Punk alors qu'à Chambéry j' allais aux gâteaux chez les mamans à 4 heures de l'après-midi. Ça forge un caractère interculturel, parce t'es obligé de t'adapter... je suis éduqué pour le changement parce que je suis formé depuis petit. Tu apprendrais à ton enfant à manger des vers de terre, dans un bol de terre, crus avec un peu de vinaigre à l'âge de un ans, à l'age de 30 ans il trouvera ça normal... c'est pour ça que j'ai amené mes enfants, ma fille de 3 ans et demi et de 7 ans, en Turquie à quatre mois parce que je voulais qu'ils sachent c'est quoi l'étonnement quand on vit chez l'autre et aujourd' hui ils n'ont pas les mêmes réflexions que le Français moyen sur l'intégration, les peurs, l'immigration et catera ».

Nos entretiens nous conduisent à formuler une hypothèse que seule une enquête plus poussée pourrait valider : la courbe tendancielle du taux de déplaisir du cadre au travail augmente avec la suractivité vécue (quand les rythmes sont particulièrement serrés et que la « sur-circulation » dans l'espace s'accroît), alors même que la mobilité géographique répétée est considérée par les entreprises comme un processus qui permet d'accroître les capacités d'adaptation du salarié.

Plus des trois-quarts des personnes interviewées évoquent des troubles liés aux tensions entre adaptation continuelle et permanence de soi, entre liberté et dépendance : "quand tu voyages, tu es complètement indépendant, tu es seul, tu n'es pas en sécurité. S'il t'arrive quelque chose et tu ne peux appeler personne, tu dois te renforcer, il y a des moments dans lesquels tu n'as pas envie d'avoir tous tes sens complètement en alerte et tu peux respirer, tu peux respirer parce que tu es en sécurité »(chef de projet multinationale 2). Les coûts touchent souvent à la sphère familiale : "Je partage la vie avec quelqu'un qui est très mobile aussi, mais j'espère un lendemain pouvoir créer une famille, alors après on arrête les deux, si on veut faire une famille ce n' est pas possible comme ça, c'est clair ça ne marcherait pas » (chef de projet, multinationale 1).

Pour ces cadres, un des enjeux revient à savoir lier réussite matrimoniale et familiale et contraintes de travail, quand précisément celles-ci entre en opposition avec celle au travail : "Ma mobilité est aussi familiale! Je me suis marié et divorcé une première fois car ma femme n'était pas mobile, elle voulait faire carrière en France et ne m'a pas suivi en Irlande, elle est très immobile, elle a toujours vécu dans les trois mêmes kilomètres carrées, on est les meilleurs amis. Elle est ingénieur, elle a fait une très belle carrière chez $x$, il faut dire qu'elle était ultrasédentaire et moi ultramobile, je me suis remarié j'ai eu deux autres enfants et je viens de divorcer pour la deuxième fois » (Cadre expatrié, multinationale 3). La mobilité géographique, pour les personnes 
étudiées, a engendré souvent une situation dissymétrique qui conduit à une division familiale du travail entre partenaires (Barrere-Maurisson, 1992). Les périodes de vie commune sont vouées, par exemple, à compenser, en accéléré, les manques ressentis pendant les mois précédents de forte mobilité.

\subsection{Ressources internationales et fatigue des top managers?}

"Je pense qu'il convient de faire une différence entre ma mobilité actuelle et ma mobilité quand j'étais un jeune cadre, mais aussi entre ma mobilité en tant que directeur et la mobilité, par exemple, d'un cadre international » (Direction générale, multinationale 1).

\subsubsection{Configuration « spatio-temporelle » 4 : Circulation du top management à mobilité interne/externe}

Alors que le management intermédiaire (configurations 1, 2 et 3 de notre enquête), comme nous l'avons vu, pointe des coûts humains de la mobilité en insistant sur l'adaptation à des cultures différentes de celles du pays d'origine, à des logiques d'un « entre-deux culturel » liées à la mobilité géographique, l'articulation des coûts que le top management nous signale est d'un autre ordre et évoque plutôt la charge physique et psychologique. "Je fais toujours des voyages plutôt courts de façon à pouvoir faire un aller-retour en faisant le maximum de rendez vous dans le même temps, donc en partant très tôt le matin, en rentrant très tard le soir pour avoir le travail maximum de la journée en passant éventuellement une nuit ou deux nuit sur place (...) c'est une vie de déplacement permanent, donc de décalage horaire permanent aussi. On est constamment sollicité et l'on n'arrive pas à se reposer vraiment. Résultat : on est fatigués en permanence (...) Il y a des gens qui effectivement prennent des tranquillisants et des excitants pour rester efficaces, ou bien quelque drink de plus »(Direction Commerciale multinationale 1). Cet extrait d'entretien illustre une configuration «spatio-temporelle » internationale des top managers que nous avons rencontré, faite de déplacements de très brève durée — de un ou deux jours - et réguliers - de l'ordre de un à trois déplacements par semaine. Cette mobilité, faite de déplacements brefs et fréquents, semble ne pas obliger à « un travail de repositionnement professionnel imposé par une migration ou un déménagement » (Kaufmann, $2005: 124)$.

La circulation du top management vise à concilier deux exigences opposées, l'exigence de "ne pas laisser le siège pour trop longtemps », et celle de constituer ou de renforcer sans cesse des partenariats avec des organisations ou des individus à l'extérieur de l'entreprise. La direction principale des déplacements est donc du siège aux filiales et lieux de réunions en interne, et aux partenaires extérieurs (et retour), d'où le nom de mobilité interne/externe (que ces partenaires extérieurs soient des clients, des fournisseurs, des actionnaires, des consultants, des journalistes, d'autres dirigeants, des chasseurs de têtes, des coachs)... 
À la fatigue qui suit le rythme des déplacements, s'ajoute, pour les « top managers », la fatigue générée par la gestion serrée des temps pendant les déplacements : "Je vous donne un exemple, on débarque aux États Unis le mercredi soir, on arrive à 19 heures on est fatigués on essaie de tenir jusqu'à 10 heures, le lendemain matin à 8 heures on commence des rendez vous toute la journée, on termine à 18 heures, et le soir quand on rentre à l' hôtel on doit reprendre le travail, on a la centaine de mails qui arrivent, on recommence le vendredi toute la journée et dès qu'on termine on prend l'avion pour être à Paris le samedi matin » (Direction Communication multinationale 2). Pour la plupart des top managers fait sens la nécessité d'être connectés en permanence pendant les déplacements. "Quand on arrive quelque part on sort de l'avion on branche le téléphone, on branche le blackberry et on reçoit ses mails, donc on est connecté en permanence et évidemment le flux de mails qui est un flux poussé augmente encore le stress, car on peut voir les mails qui arrivent, on a des réponses à faire et tout ça » (Direction Générale multinationale 3).

Être connecté en permanence signifie en fait " contrôler les subordonnés aussi bien qu'être contrôlé sans arrêt : moi, quand je suis loin du siège j'ai au moins 10 personnes qui savent ce que je suis en train de faire » (Direction Générale multinationale 2).

Plus de la moitié du middle management et trois-quarts des top managers que nous avons rencontré dénoncent une fragilisation de la sphère familiale à laquelle conduit pour eux la forme de mobilité qu'ils vivent comme le souci de ne pas voir grandir ses enfants (signalé par la majorité du top management et qui diffère du souci de "transplanter " ses enfants là où l'on est envoyé). De même, négocier avec son conjoint (les effectifs mobiles de notre enquête sont presque tous des hommes) un déplacement sur une durée de plusieurs années (configurations 1-3) n'est pas la même chose que négocier, quand il y a une négociation, des temps importants d'absences, qui diffèrent à leur tour en ce qui concerne les rythmes (conf. 4 et 5). Ces temps d'absences se combinent, notamment pour le top management avec une indisponibilité constante : "Certains ont envie d' un peu de liberté, d'être joignable pour les enfants ou pour sa femme, mais souvent ce n'est pas le moment, c'est-à-dire parce qu'on a une réunion, parce que on a un rendez-vous important, parce qu'on doit donner des ordres en ce moment (...) c'est dur pour tous" (Senior Vc Présidente multinationale 1).

Le type de mobilité fonctionnelle du top management ne semble pas déboucher sur des possibilités d'enracinement comme pour certains expatriés (au moment de la retraite notamment, configuration 1) compte tenu des temps extrêmement brefs de permanences à l'étranger ainsi que de l'emploi du temps serré. "Nous tous avons un seuil, on ne peut pas enregistrer plus d'un certain nombre d'informations par heure, par jour ou par semaine» (Direction Générale multinationale 3 ).

"Nous fréquentons en général des lieux connus à l'avance, fonctionnels, comme les hôtels proches des aéroports ou des sièges du business ». En cela, ces personnes n'ont rien de l'explorateur culturel qui se " promène », « se perd », « découvre » la ville étrangère au fil de ses intuitions. Le temps est compté. "Notre 
quotidien est aussi de plus en plus soumis aux effets des mouvements de normalisation et de standardisation comme les normes Iso, l'organisation en projet, les normes comptables internationales... Ces dispositifs de gestion produisent un espace de travail cohérent quel que soit les pays » (Direction Générale multinationale 3) (Dudouet, Mercier et Vion, 2006 : p. 367).

Ces éléments nous conduisent à distinguer top management et élites patronales qui partagent « des compétences internationales, des carnets d'adresses et des savoirfaire spécifiques » et en font « un petit milieu de dirigeants cosmopolites inscrits dans des champs différents, qui partagent des catégories de perceptions communes, au-delà de la diversité apparente des pratiques professionnelles ou des conceptions idéologiques » (Wagner, $2007: 55)$. Être cadre supérieur ne dit rien sur l'appartenance ou non à ce groupe social, à ces communautés patronales relativement soudées permettant de franchir des distances géographiques importantes ne mettant en jeu que des distances sociales faibles (Wagner, 2007 : 49). D'autres critères entrent en jeu, dont les liens familiaux et les réseaux amicaux et professionnels (Pinçon et Pinçon-Charlot, 2007 : 276). Nous distinguons donc ici «capital cosmopolite » des sphères patronales et « ressources internationales » du top management. Cela revient aussi à souligner le cosmopolitisme des vieilles familles, qui n'est certes pas une nouveauté historique et qui ne s'acquiert que par naissance ou par cooptation, alors que certaines ressources internationales en entreprise peuvent être acquises dans les «bonnes écoles » et leurs associations d'anciens élèves.

Au plus haut des grandes entreprises, observons-le, le pouvoir reste détenu par des nationaux, passés par les filières nationales même si les systèmes de cooptation nationaux pour les conseils d'administration se voient contestés par les acteurs financiers. «En France », remarque Wagner, « $43 \%$ des administrateurs des sociétés cotées en Bourse sont diplômés de Polytechnique ou de l'ENA et $70 \%$ des participations croisées sont le fait d'administrateurs issus de ces deux écoles, qui incarnent par excellence l'État et le national » (Wagner, 2008 : 42). Si plus des quatrecinquième des grands patrons allemands, britanniques, français, américains à la fin des années 90 n'avaient pas vécu dans un pays étranger, que ce soit dans leurs études ou leurs carrières (Hartmann, 1999), c'est pourtant dans la fraction la plus traditionnelle de l'aristocratie et de la grande bourgeoisie que l'on trouve les futures élites qui acquièrent les traits d'une classe transnationale de dirigeants et de jeunes managers armés pour l'exercice du pouvoir en entreprises dans la mondialisation. Au plus haut des vieilles familles d'affaire, la légitimité internationale vient s'ajouter à l'excellence nationale. À l'inverse du milieu de la population, qui doit souvent les dissoudre, ces ressources ne sont pas antinomiques mais cumulatives ${ }^{9}$.

9 «Dans la vie sociale, la valeur des richesses est le plus souvent accrue par l'ancienneté de leur acquisition. Le cosmopolitisme n'échappe pas à cette logique : le temps confère l'assurance, le savoir-faire et le savoir-être qui distinguent les héritiers des "nouveaux riches". Il contribue à légitimer les privilèges qui semblent s'inscrire "naturellement" dans l'identité sociale. La mondialisation ne subvertit donc pas les hiérarchies sociales. Mais elle a obligé les classes dominantes à adapter leurs stratégies au nouvel espace de concurrence internationale qu'elle a créé, et aux nouveaux principes d'excellence qui le régissent » (Wagner, $2008: 43$ ). 
Ces quelques éléments invitent à revenir sur le temps de structuration d'une culture internationale ou transnationale des entreprises françaises qui n'est pas une culture « en soi » mais une période de rapport où les attributs nationaux, les ressources éducatives, familiales, communautaires, doivent être mobilisées dans un jeu de mise en présence avec les trajectoires et identités professionnelles.

\subsection{Aspects conclusifs}

La valorisation croissante de la mobilité comme ressource, pendant les dernières décennies, a renforcé l'exigence de devoir " bouger » pour faire carrière au sein des couches sociales supérieures. Elle prend les traits d'un système de valeur, d'un impératif et d'une épreuve.

Dans les trois grandes entreprises que nous avons pu étudier, force est de constater qu'il n'existe pas un mais des types de cadres et dirigeants mobiles, différents en leurs conditions d'exercice professionnels comme de leurs ressources. Il n'existe pas une seule mobilité géographique renvoyant à un seul de type de compétences et de ressources acquises, ni une manière égale pour tout le monde de la vivre. Envisager la mobilité en termes de configuration «spatio-temporelles » nous a permis de retrouver des liens entre l'activité des sujets, les directions des déplacements et les exigences de l'entreprise ${ }^{10}$. Cela nous a aussi permis de comprendre les articulations de coûts que les différentes formes de mobilité que nous avons ébauchées font peser sur les sujets.

Dans nos enquêtes, les salariés mobiles sont contraints à se déplacer d'un monde social et professionnel à un autre et ils sont confrontés à une pluralité d'ordres normatifs différents les uns des autres (Roulleau-Berger2007 : 135). La réalité « multiculturelle » des grandes entreprises amène à considérer l'existence simultanée de représentations contradictoires, de pratiques de groupes de plus en plus variés et il y a fort à penser qu'à mesure que nous étudierons des mondes interconnectés (fruits de mobilités internationales du personnel, de rapports inégalitaires entre siège(s) et filiales, entre filiales entre elles...) s'enrichira une démarche scientifique davantage dynamique du sujet-travailleur, incorporant des éléments dialogiques, sémantiques et pragmatiques de l'expression des trajectoires et identités professionnelles. Si cette démarche scientifique s'oppose à l'idée de salariés mobiles identiques, sortes de citoyens du monde partout adaptable, nous n'ignorons pas l'importance d'autre type de mobilités que la mobilité géographique.

10 Les consultants en optimisation de voyages parlent de «travel pattern » pour lier les raisons des voyages et leurs directions : "La plupart du temps, la multinationale cliente nous demande de faire des questionnaires pour comprendre pourquoi les gens voyageaient et quelle est la part d' externe ou d'interne afin de mieux comprendre le "travel pattern", c'est à dire le périmètre de voyage. Où est-ce que les gens vont? Ça c'est dans les données de la multinationale. Ils le savent déjà, mais pourquoi ils y vont c'est vachement important parce que, après, tu peux décider de couper ou pas »(Consultante en optimisation des voyages d'affaires). 
De prochains travaux pourront permettre ainsi de cerner les ressources de personnes qui cumulent simultanément plusieurs charges, et qui ont donc le don d'une certaine ubiquité sociale que d'autres n'ont pas. Celui de pouvoir occuper des positions de pouvoir dans des temps qui ne sont pas simplement successifs ${ }^{11}$. C'est le cas, par exemple, dans nos enquêtes, d'un «top manager » qui travaille pour la multinationale 1 et qui est dans le conseil d'administration d'une filiale de la multinationale 2. Là où une partie du management intermédiaire apparaît comme soumis à l'obligation de s'enraciner à nouveau en terre étrangère le temps d'une expatriation pour accumuler du pouvoir, pour un temps social relativement long, là où les sphères du patronat souligne une permanence de liens sociaux à une échelle mondialisée, le top management des trois multinationales étudiées, qui transite pour un temps très limité dans des espaces fonctionnels comme les hôtels proches des aéroports et les bureaux, illustre une forme de mobilité différente de par ses rythmes, logiques et destinations. Une forme de mobilité «multipositionnelle » qui nous invite à penser que les inégalités sociales et les différentiels de pouvoir se manifestent aussi dans d'autres espaces, des espaces superposés, enchevêtrés, et qu'il ne faut pas surestimer la capacité prédictive de la mobilité géographique en tant que facteur de stratification sociale tout en soulignant son importance.

\section{Références bibliographiques}

AGARD Jean Yves (2004) Ethnographie de la mobilité internationale : le cas d'une multinationale française, Thèse de doctorat de sociologie, Sorbonne : Université René Descartes Paris 5.

BARRERE-MAURISSON Marie Agnès (1992) La division familiale du travail. La vie en double, PUF.

BERTAUX-WIAME Isabelle et TRIPIER Pierre (2006) «Les intermittents du foyer ou les arrangements entre membres des couples qui travaillent loin de l'autre » introduction au dossier, Cahiers du Genre, 41, p. 11-22.

BERTHOIN ANTAL Ariane (2000) "Types of Knowledge Gained by Expatriate Managers", Journal of General Management, 26/2, p. 32-51.

BIRKINSHAW Julian \& HOOD Neil (1998) Multinational Corporate Evolution and Subsidiary Development, Basingstoke, Macmillan.

BLACK John Stewart \& GREGERSEN Hal B. (1999) "The Right Way to Manage Expats", Harvard Business Review, 77/2, p. 52-63.

BOLTANSKI Luc et CHIAPELLO Eve (1999) Le Nouvel Esprit du Capitalisme, Gallimard.

BOLTANSKI Luc (1973) «L'espace positionnel. Multiplicité des positions institutionnelles et habitus de classe », Revue Française de Sociologie, Vol. 14, No. 1, pp. 3-26.

CERDIN Jean-Luc (2007) S'expatrier en toute connaissance de cause, Eyrolles.

COHEN Joëlle (2005) « Théories constructivistes de l'apprentissage et apprentissage collaboratif à distance»,C@rrefours télématiques.

11 Luc Boltanski a distingué les «monopositionnels» des «multipositionnels » qui, en occupant simultanément des positions institutionnelles différentes jouissent d'une forme d'ubiquité sur cet espace (1973:3-26). 
DE BETTIGNIES Henri Claude \& EVANS Paul (1977) The Cultural dimension of top executives careers: a comparative Analysis, Penguin Books.

DUDOUET François Xavier, MERCIER Delphine et VION Antoine (2006) « Les politiques internationales de normalisation », Revue Française de Science Politique, Vol. 56, $\mathrm{n}^{\circ} 3$ p. 367-392.

GIACCARDI Chiara et MAGATTI Mauro (2003) L'Io globale, Laterza.

GUPTA Anil K \& GOVINDARAJAN Vince (2000) 'Knowledge Flows within Multinational Corporations', Strategic Management Journal, 21, pp. 473-96.

HARTMANN Manfred (1999) “Auf dem Weg zur transnationalen Bourgeoisie ? Die Internationalisierung der Wirtschaft und die Internationalität der Spitzenmanager Deutschlands, Frankreichs, Grossbritanniens und der USA", Leviathan, vol. XXVII, cité par WAGNER, Anne Catherine (2008), «Élites, une classe internationale », Sciences humaines, n 191, p. 42.

HARVEY Daniel (1989) The condition of postmodernity, Blackwell Oxford University Press.

HARZING Anne Wil (2001) "Of Bears, Bumble Bees, and Spiders: The Role of Expatriates in Controlling Foreign Subsidiaries', Journal of World Business, 38(4), p. 366-379.

HEDLUND Garrett (1994) 'A Model of Knowledge Management and the N-Form Corporation', Strategic Management Journal, 15(special issue), p. 73-90.

KAUFMANN Vincent (2005) "Mobilités et réversibilités : vers des sociétés plus fluides ?», Cahiers Internationaux de Sociologie, Vol. CXVIII.

KEALEY Daniel J. (1990) L'efficacité interculturelle. Une étude des conseillers techniques canadiens à l'étranger, Agence canadienne de développement international, Hull.

MARTIN Dominique, METZGER Jean-Luc et PIERRE Philippe (2003) Les métamorphoses du monde. Sociologie de la mondialisation, Le Seuil.

PIERRE Philippe (2003) «Mobilité internationale des élites et stratégies de l'identité », Revue Européenne des Migrations Internationales, volume 19, numéro 1.

PIERRE Philippe (2003) Mobilité internationale et identités des cadres. De l'usage de l'ethnicité dans l'entreprise mondialisée, Sides.

PIERRE Philippe (2005) «L'ethnicité, ressource identitaire dans l'entreprise mondialisée. L'exemple de cadres de l'industrie pétrolière ", in Sous la direction de VINSONNEAU G., Contextes pluriculturels et identités : recherches actuelles en psychologie sociale, Éditions Sides.

PINÇON Monique et PINÇON-CHARLOT Michel (2007) Les ghettos du gotha, Le Seuil.

RAMOS Elsa (2006), L'invention des origines. Sociologie de l'ancrage identitaire, A. Colin.

ROULLEAU-BERGER Laurence (2007) « Grammaires de la reconnaissance, individuation et ordres sociétaux », in sous la direction de CAILLE Albane (2007), La quête de reconnaissance, La découverte.

SCHMID Stefan (2006) «Pas de stratégie internationale sans dirigeants internationaux », Cahiers de recherche ESCP-EAP, $\mathrm{n}^{\circ} 2$.

SAND George, Lettres d'un voyageur, A. Herbert, citée par GEOFFROY Christine, (2005) L'immigration des Britanniques à Chamonix, Colloque « La sociabilité dans tous ses états », CRIDAF.

SCHWEIGER David, M. ATAMER Tugrul et CALORI Roland (2003) 'Transnational Project Teams and Networks : Making the Multinational More Effective', Journal of World Business, 38, pp. 127-140.

URRY John (2000) Sociology beyond societies, Routledge.

VIGNAL Cécile (2005) «Injonctions à la mobilité, arbitrages résidentiels et délocalisation de l'emploi », Cahiers internationaux de sociologie, vol.CXVIII.

WAGNER Anne Catherine (2003) «La bourgeoisie face à la mondialisation », Mouvements, $\mathrm{n}^{\circ} 26$. 
WAGNER Anne Catherine (2008) «Élites, une classe internationale », Sciences humaines, $\mathrm{n}^{\circ} 191$.

WAGNER, Anne Catherine (1998) Les nouvelles élites de la mondialisation. Une immigrée dorée en France, PUF.

WAGNER, Anne Catherine (2007) Les classes sociales dans la mondialisation, La Découverte.

WIHTOL DE WENDEN Catherine (2001) « Un essai de typologie des nouvelles mobilités », Hommes \& Migrations, $\mathrm{n}^{\circ} 1233$, p. 5-12. 
